\title{
Role of glycemic control on hospital-related outcomes in patients with diabetes mellitus undergoing renal transplantation
}

This article was published in the following Dove Press journal:

Diabetes, Metabolic Syndrome and Obesity:Targets and Therapy

5 January 2017

Number of times this article has been viewed

\author{
Elizabeth M Lamos' \\ Marniker A Wijesinha ${ }^{2}$ \\ Seba Ramhmdani' \\ Laurence S Magder ${ }^{2}$ \\ Kristi D Silver \\ 'Division of Endocrinology, Diabetes \\ and Nutrition, ${ }^{2}$ Department of \\ Epidemiology, University of Maryland \\ School of Medicine, Baltimore, \\ MD, USA
}

Objective: To compare length of stay (LOS) and incidence of hypoglycemic events and infections in hospitalized patients with diabetes mellitus (DM) undergoing renal transplantation, among groups of patients defined by admission glucose and mean inpatient daily glucose.

Methods: A retrospective analysis of 190 charts of patients with DM who underwent renal transplantation over a 2-year period was conducted. Patients were grouped according to admission glucose and mean inpatient daily glucose ( $\leq 140 \mathrm{mg} / \mathrm{dL}, 141-180 \mathrm{mg} / \mathrm{dL}$, and >180 mg/dL). Results: Admission glucose was not associated with LOS. A mean inpatient daily glucose of $\leq 140 \mathrm{mg} / \mathrm{dL}$ was associated with a longer LOS compared to a mean inpatient daily glucose of $>180 \mathrm{mg} / \mathrm{dL}(p=0.03)$. Patients with an admission glucose of $\leq 140 \mathrm{mg} / \mathrm{dL}$ had approximately half the rate of hypoglycemic events compared to those with admission glucose of 141-180 mg/dL (odds ratio $[\mathrm{OR}]=2.1 ; p=0.02)$ or $>180 \mathrm{mg} / \mathrm{dL}(\mathrm{OR}=1.9 ; p=0.04)$. However, patients whose mean daily glucose was $\leq 140 \mathrm{mg} / \mathrm{dL}$ had approximately twice the rate of hypoglycemic events than those whose mean daily glucose was $141-180 \mathrm{mg} / \mathrm{dL}(\mathrm{OR}=0.4 ; p=0.01)$ or $>180 \mathrm{mg} / \mathrm{dL}$ $(\mathrm{OR}=0.4 ; p=0.004)$. The incidence of infections was low and was not associated with admission or mean daily glucose levels.

Conclusion: Lower mean daily inpatient glucose levels $(\leq 140 \mathrm{mg} / \mathrm{dL})$ are associated with longer LOS and greater incidence of hypoglycemic episodes in diabetes patients undergoing renal transplantation. Our findings suggest that target blood glucose levels of 140-180 mg/dL may be appropriate in this specific population. Additional prospective research is needed to confirm these findings.

Keywords: diabetes mellitus, renal transplantation, length of stay, hypoglycemia, infection

\section{Introduction}

Diabetes mellitus (DM) is the leading cause of end-stage renal failure, requiring hemodialysis and renal transplantation, in the US. ${ }^{1}$ Classic markers of renal transplantation success are based on graft survival, patient morbidity including immunosuppressive toxicity and infection, and patient mortality. ${ }^{2}$ Diabetic patients who undergo renal transplantation have an increased risk of infection, rehospitalization, and inpatient mortality. ${ }^{3,4}$ Hyperglycemia has been linked to worse endothelial function, impaired wound healing, and pro-inflammatory conditions, ${ }^{5}$ which have been postulated to partially explain the association of preexisting DM with poor clinical transplant outcomes. ${ }^{6}$ Thus, targeting inpatient hyperglycemia management in the perioperative period in diabetic patients undergoing renal transplant could improve morbidity in this population. However, there is a paucity of published data on this topic. This is in contrast to the many studies evaluating glycemic control in cardiac, trauma, and intensive care unit
Correspondence: Kristi D Silver Division of Endocrinology, Diabetes and Nutrition, University of Maryland School of Medicine, 660 West Redwood Street, $\mathrm{HH} 494$, Baltimore, MD $2120 \mathrm{I}$, USA

Tel + I 4107061628

Fax +I 4I0 7061622

Email ksilver@medicine.umaryland.edu 
patients, ${ }^{7-9}$ which has led to debate regarding the glycemic targets for hospitalized patients.

The aim of this study was to evaluate patients with DM who underwent renal transplantation and compare the clinical outcomes of length of stay (LOS), hypoglycemic events, and infections rates among patient groups defined by admission glucose and mean inpatient daily glucose levels (a measure of inpatient glycemic control).

\section{Research design and methods}

A single-center retrospective chart review was conducted of adult patients (age $\geq 18$ years) with a known diagnosis of DM, defined using the International Classification of Diseases, Ninth Revision (ICD-9) code 250.xx and medical record documentation indicating a diagnosis of DM, who underwent renal transplantation at the University of Maryland Medical Center from December 23, 2008 to December 23, 2010. Patients who received dual solid organ transplantation at the time of or prior to the kidney transplant were excluded. Two hundred and eight patients were included in the study based on inclusion and exclusion criteria.

Admission plasma glucose was defined as the first recorded plasma glucose level at hospital admission for renal transplantation. Capillary glucose measurements were obtained using a Roche AccuChek Inform glucometer (Roche Diagnostics, Indianapolis, IN, USA). Only patients with a minimum of three capillary glucose results each day were included in this analysis. The mean daily glucose level was calculated by taking the average of the patient's mean glucose values for each of the days on which glucose was measured during their hospital stay. Patients were stratified into three groups based on their admission plasma glucose and mean daily glucose levels: $\leq 140 \mathrm{mg} / \mathrm{dL}, 141-180 \mathrm{mg} /$ $\mathrm{dL}$, and $>180 \mathrm{mg} / \mathrm{dL}$ in order to compare the three outcome variables of interest: LOS, rate of hypoglycemic events (defined as a blood glucose level $<70 \mathrm{mg} / \mathrm{dL}$ ), and rate of infections. Blood glucose stratification was chosen based on current inpatient blood glucose targets. ${ }^{10}$ The relationship between the categorical variables, such as sex, age, race, type of renal transplant, and Charlson comorbidity index score, ${ }^{11}$ and the outcome variables of interest was also examined. The statistical significance of differences between the subgroups with respect to mean LOS was calculated based on an analysis of variance model. Renal function was defined as glomerular filtration rate (GFR) on the modification of diet in renal disease equation and was calculated using the serum creatinine levels and the patient's age, race, and sex on the day of discharge or at day 7 of hospitalization using whichever was shorter. As patients were followed for variable lengths of time, a discrete-time survival analysis was performed for the incidence of hypoglycemic events. Only the first hypoglycemic event per person was considered for this analysis. For all tests, a $p$-value $<0.05$ was considered significant. This study was approved by the Institutional Review Board of the University of Maryland, Baltimore which waived the requirement to obtain patient's consent due to the retrospective nature of the study.

\section{Results}

Of the 214 patients identified with diabetes who underwent renal transplantation, 24 patients were excluded from the study due to incomplete data or other solid organ transplants. One individual was excluded from the LOS calculation as the LOS was more than three times the standard deviation for LOS. Among the 190 individuals included in the study, $67 \%$ were male, $52 \%$ were black, and $87 \%$ were older than 45 years of age (mean, $58 \pm 11.5$ years) at the time of cohort entry (Table 1). Eighty-eight percent had a diagnosis of type $2 \mathrm{DM}$, and $12 \%$ had a diagnosis of type $1 \mathrm{DM}$. Most patients (77\%) underwent a deceased donor renal transplantation. Sixty-three percent of patients in the deceased donor (DD) category had delayed graft function, while none in the living donor (LD) and living related donor (LRD) categories reported delayed graft function.

The mean LOS was $8.0 \pm 4.5$ days (Table 1 ). Those patients whose mean daily glucose was $\leq 140 \mathrm{mg} / \mathrm{dL}$ had a longer LOS (9.9 \pm 6.1 days) compared to those with a mean daily glucose of $>180 \mathrm{mg} / \mathrm{dL}(7.5 \pm 3.6$ days, $p=0.03)$. The LOS for patients with a mean daily glucose of $141-180 \mathrm{mg} / \mathrm{dL}$ was similar to that of the patients with $\leq 140 \mathrm{mg} / \mathrm{dL}(8.6 \pm 5.4$ days, $p=0.4$ ). Further stratification within the group with an average daily glucose of $\leq 140 \mathrm{mg} / \mathrm{dL}$ did not demonstrate any statistical difference in LOS (data not shown). Admission glucose was not associated with LOS. The mean LOS among the 177 patients not experiencing acute rejection was $7.9 \pm 4.3$ days, compared to $10.9 \pm 7.4$ days among the 13 patients experiencing acute rejection $(p=0.2)$; thus, acute rejection did not significantly increase LOS.

Table 1 shows the relationship between patient characteristics and the incidence of hypoglycemic events. Forty-four percent of patients had at least one hypoglycemic event. The overall hypoglycemia incidence rate was 0.084 events per person-day. Deceased donor renal transplantation was associated with a lower rate of hypoglycemic events compared to LD renal transplantation $(p=0.03)$. Age was not associated with rate of hypoglycemic events. Patients with an admission glucose of $\leq 140 \mathrm{mg} / \mathrm{dL}$ had approximately half the rate of hypoglycemic events compared to those with an admission 
Table I Comparison of variables with length of stay and hypoglycemic events

\begin{tabular}{|c|c|c|c|c|c|c|}
\hline Variables & Patients & Mean LOS (days) & $p$-Value & Hypoglycemia rate per person-day & OR & $p$-Value \\
\hline \multicolumn{7}{|l|}{ Sex } \\
\hline Male & 128 & 7.6 & $\mathrm{R}$ & 0.082 & $\mathrm{R}$ & \\
\hline Female & 62 & 8.9 & 0.08 & 0.090 & I.I & 0.66 \\
\hline \multicolumn{7}{|c|}{ Age group (years) } \\
\hline $18-34$ & 4 & 8.3 & 1.00 & 0.032 & 0.4 & 0.34 \\
\hline $35-44$ & 21 & 8.3 & 1.00 & 0.068 & 0.8 & 0.90 \\
\hline $45-59$ & 73 & 7.6 & 0.72 & 0.099 & 1.3 & 0.13 \\
\hline$\geq 60$ & 92 & 8.3 & $\mathrm{R}$ & 0.079 & $\mathrm{R}$ & \\
\hline \multicolumn{7}{|l|}{ Race } \\
\hline White & 81 & 8.0 & $\mathrm{R}$ & 0.088 & $\mathrm{R}$ & \\
\hline Black & 98 & 8.3 & 0.86 & 0.079 & 0.9 & 0.34 \\
\hline Other & 11 & 6.5 & 0.54 & 0.119 & 1.4 & 0.42 \\
\hline \multicolumn{7}{|l|}{ Organ } \\
\hline Kidney DD & 145 & 8.5 & 0.01 & 0.074 & 0.5 & 0.03 \\
\hline Kidney LD & 33 & 6.2 & $\mathrm{R}$ & 0.130 & $\mathrm{R}$ & \\
\hline Kidney LRD & 12 & 7.6 & 0.54 & 0.133 & 1.0 & 0.46 \\
\hline \multicolumn{7}{|c|}{ Charlson score } \\
\hline 0 & 4 & 9.0 & $\mathrm{R}$ & 0.032 & 0.7 & 0.99 \\
\hline I & 1 & 4.0 & 0.61 & 0.000 & $N / A$ & 0.98 \\
\hline 2 & 110 & 8.5 & 0.99 & 0.098 & 2.4 & 0.97 \\
\hline 3 & 61 & 7.2 & 0.76 & 0.079 & 1.9 & 0.98 \\
\hline 4 & 14 & 8.4 & 0.99 & 0.043 & $\mathrm{R}$ & \\
\hline \multicolumn{7}{|c|}{ Admission glucose (mg/dL) } \\
\hline$\leq 140$ & 124 & 8.0 & $\mathrm{R}$ & 0.069 & $\mathrm{R}$ & \\
\hline $14 \mid-180$ & 22 & 8.1 & 0.999 & 0.137 & 2.1 & 0.02 \\
\hline$>180$ & 28 & 8.2 & 0.98 & 0.121 & 1.9 & 0.04 \\
\hline \multicolumn{7}{|c|}{ Mean daily glucose (mg/dL) } \\
\hline$\leq 140$ & 24 & 9.9 & $\mathrm{R}$ & 0.168 & $\mathrm{R}$ & \\
\hline$|4|-180$ & 45 & 8.6 & 0.38 & 0.076 & 0.4 & 0.01 \\
\hline$>180$ & 119 & 7.5 & 0.03 & 0.076 & 0.4 & 0.004 \\
\hline
\end{tabular}

Abbreviations: DD, deceased donor; LD, living donor; LOS, length of stay; LRD, living related donor; OR, odds ratio; R, reference; N/A, not applicable.

glucose of $141-180 \mathrm{mg} / \mathrm{dL}(p=0.02)$ or $>180 \mathrm{mg} / \mathrm{dL}(p=0.04$; Table 1). However, patients with a mean inpatient daily glucose of $\leq 140 \mathrm{mg} / \mathrm{dL}$ had approximately twice the rate of hypoglycemic events compared to those with a mean daily glucose of $141-180 \mathrm{mg} / \mathrm{dL}$ or $>180 \mathrm{mg} / \mathrm{dL}$ ( $p=0.01$ and 0.004 , respectively). When stratified by donor category (DD, LD, LRD), having a mean daily glucose of $\leq 140 \mathrm{mg} / \mathrm{dL}$ was associated with significantly higher rates of hypoglycemia in the DD group only. A comparison of the incidence of hypoglycemia among donor groups was not significantly different; however, this is likely due to the small numbers in the LD and LRD groups.

Overall, the mean daily glucose did not have a statistically significant association with renal function, which was defined by automated GFR values $\left(\mathrm{mL} / \mathrm{min} / 1.73 \mathrm{~m}^{2} ; p=0.60\right.$; data not shown). Although the association was not statistically significant, there was a weak trend that patients with a lower mean daily glucose level $(\leq 140 \mathrm{mg} / \mathrm{dL})$ were more likely to have a lower GFR.

Twenty-three patients had at least one urinary tract infection. Females had a higher rate of urinary tract infections $(p=0.007)$. The incidence of urinary tract infections was not associated with age, race, type of renal transplant, hypoglycemia, mean daily glucose, or admission glucose levels. Due to the very small number of blood infections (three blood infections in 190 patients with an incidence rate of 0.002 events per person-day), no analysis was performed for blood infections. Mean daily glucose did not have a statistically significant association with the occurrence of skin or wound infections ( $p=0.2$; data not shown). The occurrence of infections (urinary tract, wound/skin, and blood infections were excluded due to low incidence) had no statistically significant associations with any variable of interest.

Charlson comorbidity index score and race were not associated with any outcome variable of interest, including sex, age, type of renal transplant, hypoglycemia, mean daily glucose, or admission glucose levels.

\section{Discussion}

The American Diabetes Association and the European Association for the Study of Diabetes have defined inpatient 
glycemic goals for critically ill patients as $140-180 \mathrm{mg} / \mathrm{dL}$ and preprandial and random glucose levels for noncritically ill patients as $<140 \mathrm{mg} / \mathrm{dL}$ and $<180 \mathrm{mg} / \mathrm{dL}$, respectively. ${ }^{10}$ Avoiding hypoglycemia is a priority, especially in the critically ill population. Some patients may be appropriate for more intensive control based on clinical judgment. Alternatively, terminally ill patients or patients with significant comorbidities may have targets higher than standard glucose ranges. ${ }^{10}$ These goals aim to reduce morbidity and mortality impacted by hyperglycemia and hypoglycemia in the inpatient population.

These guidelines fail to specifically address special populations, including high-risk diabetic patients undergoing renal transplant. Retrospective data shows a negative impact of diabetes on $\operatorname{LOS}^{12}$ and infection. ${ }^{3}$ There are conflicting data, especially, regarding graft outcomes and mortality. ${ }^{13,14}$ One prospective study by Hermayer et $\mathrm{al}^{15}(\mathrm{n}=104)$ attempted to address whether intensive blood glucose control (70-110 mg/ $\mathrm{dL}$ ) or standard blood glucose control (70-180 mg/dL), in patients with diabetes or impaired glucose tolerance, affected delayed graft function. There was no statistical difference in graft function between the two treatment groups, although severe hypoglycemia (blood glucose $<40 \mathrm{mg} / \mathrm{dL}$ ) was more common in the intensively treated group ( $16 \%$ of intensively treated patients versus $4 \%$ of control patients, $p=0.08$ ). In addition, Ramirez et $\mathrm{al}^{16}$ retrospectively evaluated 202 individuals undergoing renal transplant $(60 \%$ with preexisting diabetes). There was no association between perioperative or chronic glycemic control within the first year of postrenal transplant and graft rejection, infection, or hospital readmission even for those with the tightest glycemic control $(80-110 \mathrm{mg} / \mathrm{dL})$. Although small, these studies start to address whether diabetic renal transplant patients should be grouped with critically ill patients or with noncritically ill patients for their inpatient target glucose goals.

Our study adds to this limited body of literature. Admission glucose did not impact the clinical parameters of LOS or infection. These results are in contrast to the retrospective study by Tewari et al, ${ }^{12}$ which demonstrated that patients with an admission glucose $>180 \mathrm{mg} / \mathrm{dL}$ had an increased LOS regardless of diabetes status prior to renal transplantation. In our study, those individuals with higher admission glucose levels may have been subjected to a more aggressive glucose management plan, thus potentially explaining the increased incidence of hypoglycemic events in this group.

Patients whose daily glucose levels were more strictly controlled (mean glucose $\leq 140 \mathrm{mg} / \mathrm{dL}$ ) had longer LOS and were more likely to have a hypoglycemic event. Although no specific or consistent hyperglycemia treatment protocol was used for diabetic patients undergoing renal transplant, it is reasonable to consider that those individuals who were more strictly controlled would be expected to have more hypoglycemic events by virtue of starting at a lower overall mean glucose. Those individuals with a lower GFR, which may contribute to longer LOS, may have been more aggressively targeted for lower blood sugars because they did not have immediate graft function postoperatively. Additionally, in our study, the incidence of delayed graft function within the DD recipients compared to the LD and LRD groups was higher and may be one factor for the increased LOS in this group.

This study's findings support those observed previously that there is no significant difference between standard blood glucose control $(<180 \mathrm{mg} / \mathrm{dL})$ and stricter blood glucose control in terms of graft function or incidence of infections; $;^{16,17}$ however, stricter glucose control is associated with a greater incidence of hypoglycemic events. ${ }^{14,15}$ Collectively, these results suggest that target glucose levels for diabetic renal transplant patients should be $140-180 \mathrm{mg} / \mathrm{dL}$ while hospitalized, rather than stricter control, so that hypoglycemia may be reduced.

\section{Limitations}

As this is a retrospective study, there are several limitations. The retrospective design did not provide us the opportunity to control for multiple factors impacting glucose levels, including nutrition, insulin regimen, and immunosuppressive medications. Generally, all patients received a standard protocol for immunosuppression posttransplantation, which included high-dose glucocorticoids in combination with a calcineurin inhibitor (primarily tacrolimus) and an mammalian target of rapamycin (mTOR) inhibitor (primarily sirolumus) which are known to affect glucose homeostasis. Additionally, the chart review did not allow us to capture information on the underlying reasoning behind decisions of glucose management (e.g., allowing higher glucose levels because of hypoglycemic unawareness). LOS can be reflective of multiple variables other than those highlighted in this study, including surgical complications, rehabilitation status, and home care coordination. Thus, the contribution of any or all of these factors has not been accounted for in this study. As preadmission glycated hemoglobin results were not available for most patients, the contribution of preadmission glucose control to peritransplant glucose control cannot be defined.

\section{Conclusion}

Our results support the need for more prospective research to clarify key management issues in the diabetic renal transplant population. Glycemic control is increasingly more difficult 
when balancing variables like renal function and steroid/immunosuppressive regimens in this population. However, our study results demonstrate a snapshot of a typical "real-life" situation that adds valuable insight into the effect of glycemia on specific hospital outcomes. Intensive glucose control does not appear to reduce LOS and may contribute to hypoglycemic events.

\section{Summary}

- Post-renal transplant diabetes glycemic goals are poorly studied

- Ideal glycemic control postoperatively is difficult in this high-risk, complicated population

- Diabetic renal transplant patients should target glucose levels of $<140-180 \mathrm{mg} / \mathrm{dL}$ while hospitalized, rather than stricter control, so that hypoglycemia may be reduced

- Targeting lower blood sugars was associated with longer length of stay and increased hypoglycemia

\section{Acknowledgments}

This work was supported by American Diabetes Association grant \#1-08-CR-60 (KS). The authors would like to acknowledge Sofanit Getahun, $\mathrm{PhD}$, and Laila S Tabatabai, MD for their assistance in data collection.

Abbreviations: DD, deceased donor; DM, diabetes mellitus; GFR, glomerular filtration rate; LD, living donor; LRD, living unrelated donor; LOS, length of stay; LRD, living related donor; OR, odds ratio; ICD-9, International Classification of Diseases, Ninth Revision.

\section{Disclosure}

The authors report no conflicts of interest in this work.

\section{References}

1. Centers for Disease Control and Prevention. National diabetes fact sheet: national estimates and general information on diabetes and prediabetes in the United States; 2011. Available from: http://www.cdc.gov/diabetes/ pubs/pdf/ndfs_2011.pdf. Accessed July 2016.
2. Chapman JR. Clinical renal transplantation: where are we now, what are our key challenges? Transplant Proc. 2010;42(Suppl 9):S3-S6.

3. Lansang MC, Ma L, Schold JD, Meier-Kriesche HU, Kaplan B. The relationship between diabetes and infectious hospitalizations in renal transplant recipients. Diabetes Care. 2006;29(7):1659-1660.

4. Ramezani M, Ghoddousi K, Hashemi M, et al. Diabetes as the cause of end-stage renal disease affects the pattern of post kidney transplant rehospitalizations. Transplant Proc. 2007; 39(4):966-969.

5. Kolluru GK, Bir SC, Kevil CG. Endothelial dysfunction and diabetes: effects on angiogenesis, vascular remodeling, and wound healing. Int J Vasc Med. 2012;918267.

6. Kuo HT, Sampaio MS, Vincenti F, Bunnapradist S. Associations of pretransplant diabetes mellitus, new-onset diabetes after transplant, and acute rejection with transplant outcomes: an analysis of the Organ Procurement and Transplant Network/United Network for Organ Sharing (OPTN/UNOS) database. Am J Kidney Dis. 2010;56(6): $1127-1139$.

7. Haga KK, McClymont KL, Clarke S, et al. The effect of tight glycaemic control, during and after cardiac surgery, on patient mortality and morbidity: a systematic review and meta-analysis. J Cardiothorac Surg. 2011;6:3.

8. Gale SC, Sicoutris C, Reilly PM, Schwab CW, Gracias VH. Poor glycemic control is associated with increased mortality in critically ill trauma patients. Am Surg. 2007;73(5):454-460.

9. Dellinger RP, Levy MM, Rhodes A, et al. Surviving sepsis campaign: international guidelines for management of severe sepsis and septic shock: 2012. Crit Care Med. 2013;41(2):580-637.

10. American Diabetes Association. Standards of medical care in diabetes 2016. Diabetes Care. 2016;39(Supp1 1):S1-S108.

11. D'Hoore W, Sicotte C, Tilquin C. Risk adjustment in outcome assessment: the Charlson comorbidity index. Methods Inf Med. 1993;32(5): 382-387.

12. Tewari S, Kathuria A, Meier-Kriesche HU, Lansang MC. Association between admission hyperglycemia and length of stay after renal transplantation. Endocr Pract. 2010;16(5):805-809.

13. Rodelo JR, Nieto-Rios JF, Serna-Higuita LM, et al. Survival of renal transplantation patients older than 60 years: a single-center experience. Transplant Proc. 2013;45(4):1402-1409.

14. Rocha A, Malheiro J, Martins LS, et al. Kidney transplantation in type 2 diabetic patients: a matched survival analysis. Transplant Proc. 2013;45(6):2141-2146.

15. Hermayer KL, Egidi MF, Finch NJ, et al. A randomized controlled trial to evaluate the effect of glycemic control on renal transplantation outcomes. J Clin Endocrinol Metab. 2012;97(12):4399-4406.

16. Ramirez SC, Maaske J, Kim Y, et al. The association between glycemic control and clinical outcomes after kidney transplantation. Endocr Pract. 2014; 20(9):894-900

17. van den Berg TJ, Bogers H, Vriesendorp TM, Surachno JS, CeVries JH, ten Berge IJ, et al. No apparent impact of increased post-operative blood glucose levels on clinical outcome in kidney transplant recipients. Clin Transplant. 2009;23(2):256-263.
Diabetes, Metabolic Syndrome and Obesity: Targets and Therapy is an international, peer-reviewed open-access journal committed to the rapid publication of the latest laboratory and clinical findings in the fields of diabetes, metabolic syndrome and obesity research Original research, review, case reports, hypothesis formation, expert opinion and commentaries are all considered for publication. The manuscript management system is completely online and includes a very quick and fair peer-review system, which is all easy to use. Visit http://www.dovepress.com/testimonials.php to read real quotes from published authors. 